\title{
Adherence to intermittent preventive treatment for malaria with sulphadoxine-pyrimethamine and outcome of pregnancy among parturients in South East Nigeria
}

\author{
This article was published in the following Dove Press journal: \\ Patient Preference and Adherence \\ 07 April 2014 \\ Number of times this article has been viewed
}

\section{Azubike Kanario \\ Onyebuchi' \\ Lucky Osaheni Lawani² \\ Chukwuemeka Anthony \\ lyoke ${ }^{3}$}

Chukwudi Robinson Onoh'

Nwabunike Ekene Okeke ${ }^{4}$

'Department of Obstetrics and Gynecology, Federal Teaching Hospital, Abakaliki, Nigeria; ${ }^{2}$ School of Postgraduate Studies, Department of Community Medicine, University of Nigeria; ${ }^{3}$ Department of Obstetrics and Gynecology, University of Nigeria Teaching Hospital, Enugu, Nigeria; ${ }^{4}$ Department of Obstetrics and Gynecology, Mile Four Catholic Hospital, Abakaliki, Nigeria
Correspondence: Chukwuemeka Anthony lyoke

Department of Obstetrics and Gynaecology, University of Nigeria Teaching Hospital, Ituku-Ozalla, Enugu,

Enugu State, Nigeria

Tel +234808583 II67

Email caiyoke@yahoo.co.uk
Background: Intermittent preventive treatment of malaria for pregnant women (IPTp) is a very important strategy for the control of malaria in pregnancy in malaria-endemic tropical countries, where mosquito bites easily occur during evening outdoor activities. Issues related to provision, cost, and acceptability may affect the use of IPTp in some developing countries. The aim of the study was to assess the uptake and adherence to sulphadoxine-pyrimethamine-based intermittent preventive treatment of malaria during pregnancy and the relationship of IPTp use to pregnancy outcomes in two major obstetric centers in South East Nigeria.

Methods: This was a prospective descriptive study involving women who received antenatal and delivery services. All recruited women were followed-up from booking until delivery, and statistical analysis was done with Epi Info version 7.

Results: A total of 516 parturients were studied. The mean gestational age at booking was $21.8 \pm 6.9$ weeks while the mean number of antenatal visits throughout the pregnancy was 5.5 \pm 3.1 . The rate of uptake of at least one dose of prescribed IPTp was $72.1 \%(367 / 516)$. Of the 367 who took prescribed IPTp, adherence to second doses of IPTp was 59.7\% (219/367), and only $4.9 \%$ (18/367) took a third dose. Clinical malaria occurred in 85\% (127/149) of women who did not receive IPTp at all compared to $20.5 \%$ of those who received at least one dose of IPTp. All those who had clinical malaria despite IPTp had only one dose of IPTp despite booking in the second trimester. Malaria in pregnancy occurred significantly more in women who failed to adhere to subsequent doses of IPTp than in those who adhered $(24.6 \%$ versus $14.3 \%$, respectively; risk ratio $=2.5 ; 95 \%$ confidence interval $2.1,3.0 ; P<0.001)$. Similarly, neonatal malaria occurred significantly more in neonates whose mothers did not receive IPTp compared to those whose mothers received at least one dose of IPTp (7.4\% versus 3.4\%; risk ratio $=1.4 ; 95 \%$ confidence interval $0.9,2.1 ; P=0.003)$.

Conclusion: More than one half of parturients failed to adhere to prescribed intermittent preventive treatment for malaria in pregnancy in the major obstetric centers in Abakaliki, South East Nigeria. The very high prevalence of malaria among women who failed to adhere to IPTp and the associated adverse neonatal outcomes demands more pragmatic ways of improving access to, and acceptability of, malaria preventive measures in this area.

Keywords: malaria in pregnancy, pregnancy outcome, prevention

\section{Introduction}

Annually, women and children in sub-Sahara Africa die in large numbers from the burden of malaria. Malaria is endemic in Nigeria and has remained so despite numerous efforts by the Roll Back Malaria Partnership initiative and the United Nations 
Millennium Development Goals aimed at reducing its scourge. It is a major public health challenge, as it impedes human development. ${ }^{1}$ It is both a cause and consequence of underdevelopment and remains one of the leading causes of morbidity and mortality in Nigeria. ${ }^{1,2}$ The most vulnerable groups are children under 5 years and pregnant women., ${ }^{1,2}$

Currently, it is estimated that each year more than 125 million women become pregnant in malaria-endemic areas of the world, with 30 million of these in sub-Sahara Africa, and that 75,000-200,000 infant deaths are attributed to malaria infection in pregnancy., ${ }^{3,4}$ Reports show that the burden of malaria in pregnancy in Nigeria is high. A study by Wagbatsoma and Omoike showed that $100 \%$ of women who had no prophylaxis experienced attacks of malaria, with $76 \%$ experiencing two or more attacks. ${ }^{5}$ Similarly, a study by Chukwuocha et al found that $51.1 \%$ of pregnant women surveyed using Giemsa staining were positive for malaria infection. ${ }^{6}$ These unenviable statistics could further worsen the already poor maternal and perinatal health indices in the developing world and thus constitute a major setback to efforts in attaining the United Nations Millennium Development Goals 4, 5, and 6. ${ }^{7}$

The global policy for malaria prevention and control in pregnancy emphasizes a preventive package of IPTp, use of insecticide treated nets (ITNs), and effective case management of malaria illness and anemia., ${ }^{7,8}$ The World Health Organization (WHO) states that as early as possible in the second trimester, IPTp-sulphadoxine-pyrimethamine (SP) is recommended for all pregnant women at each scheduled antenatal care (ANC) visit until the time of delivery, provided that the doses are given at least 1 month apart. ${ }^{8}$ The drug should be administered under supervision (directly observed treatment [DOT]) during ANC visits. ${ }^{8}$ SP is currently recommended by the WHO because of its safety and efficacy in pregnancy. ${ }^{9}$

Although there have been several studies that examined the use of ITNs by pregnant women in Nigeria, ${ }^{10-13}$ few studies have evaluated the uptake or adherence to IPTp among pregnant women. Whereas bed nets are the best way to prevent mosquito bites at night, the fact that mosquitoes also bite during dusk/evening outdoor activities makes IPTp a very important component of the strategies to prevent malaria during pregnancy. This study evaluates the uptake of the initial doses and adherence to subsequent doses of IPTp during pregnancy and their relationship to pregnancy outcomes among booked parturient women in two major obstetric centres in Ebonyi State, South East Nigeria.

\section{Materials and methods}

This was a prospective observational study conducted between June 1, 2012 and December 31, 2012 in two major obstetric facilities (Federal Medical Centre, now Federal Teaching Hospital, and the Mile Four Catholic Maternity Hospital) offering ANC and delivery services to parturients. Both centers offer routine and specialized obstetric care to women living in Abakaliki, the capital city of Ebonyi State, South East Nigeria and the surrounding area. Both hospitals had a total of 4,680 deliveries during the study period.

Ethical clearance was obtained for this study from the research and ethical committees of both institutions. The study population included all pregnant women who registered for ANC in the study centers during the study period. This population was made up of urban dwellers, most of whom had formal education, as well as rural dwellers from surrounding rural communities, many of whom may have had no formal education. Most pregnant women came to these hospitals by self-referral although some women were referred from peripheral hospitals because of complicated pregnancies.

The sampling technique was purposive, and patients were enrolled consecutively. The point of recruitment was at the booking clinic. The study sample included women who booked for ANC between 14-28 weeks of gestation. Each woman recruited for the study was provide individual counseling, after which her written/oral consent was obtained. Counselling was mostly in Igbo language; women who did not understand Igbo language were counselled in English language. Each study participant was given a number (or tag) to enable specific follow-up. Study participants were followed up from booking till delivery and subsequent discharge. Women with multiple pregnancy and preeclampsia as well as medical complications of pregnancy were excluded from the study.

The procedure for ANC was similar in both study hospitals. Following booking in the antenatal clinic, women with uncomplicated pregnancies were seen subsequently every 4 weeks till 28 weeks, every two weeks till 36 weeks, and weekly till onset of labor or elective delivery. Women with complications were usually seen more frequently, depending on their conditions; alternatively, they could be admitted into the antenatal ward for in-patient care. A birth plan was formulated for every woman at the time of booking, and it could be modified as events unfolded during the prenatal period. At every antenatal visit a short history of events since their last visit was taken by the midwife, a urine dipstick test for protein, sugar, and ketones was done, and weight and blood pressure measurements were taken. 
Results of previous investigations were retrieved. All these results were documented before the woman saw the doctor, who reviewed the data and conducted a physical examination on the patient. The symphysis-fundal height, fetal lie, position, presentation, and fetal heart tones were documented. Further steps in the woman's management were then outlined. IPTp for malaria was prescribed by the doctor, and patients paid for and collected SP as prescribed. The clinics required that each pregnant woman received at least two doses of SP as IPTp during pregnancy. Women with known allergy to sulphonamides were given proguanil. Women who were enrolled on the National Health Insurance collected drugs free of charge. Participating women were all given free ITNs at booking and were encouraged to use them consistently and correctly while those who purchased IPTp or were given IPTp free of charge were encouraged to take the medication in the clinics under the observation of the nursing staff of the clinics (DOT). Women who could not participate in DOT (due to financial, time, or other constraints) were encouraged to take SP at home. Tetanus immunization was given to every pregnant woman who had not been fully immunized, based on the WHO protocol. Ultrasound scan was done as indicated. The woman was then scheduled for another appointment.

Four of the authors (OLL, AKO, OCR, and ONE) saw every participating woman in the clinics and were informed when any of the women presented for delivery. Evidence for intake of IPTp included record of DOT by a nurse or affirmation of intake of IPTp at home during questioning by the doctor. Information on compliance with IPTp in terms of initiation and follow-up doses, diagnosis of clinical malaria during the pregnancy, and delivery outcomes were recorded for each study participant during each clinic visit. Adherence to IPTp-SP was defined as intake of at least two doses of SP during the antenatal period while uptake refers to intake of at least one dose of IPTp-SP. Clinical malaria was diagnosed mainly by rapid diagnostic test for those presenting acutely and by microscopy for those with subacute symptoms. Information on labor and delivery outcome was obtained at the point of delivery. All information was recorded in a pro forma, which was developed for the study. Women with known allergy to SP and women who failed to deliver in the study hospitals were excluded from the study.

Statistical analysis was done using Epi Info statistical software, version 7.0 (Centre for Disease Control and Prevention, Atlanta, GA, USA). Descriptive statistics were obtained by means of proportions and simple percentages. The main outcome variables were the proportions of women who received the initial and subsequent doses of IPTp-SP. Inferential statistics involved estimation of risk ratios (RR) and their 95\% confidence limits for the occurrence of malaria in women who failed to adhere to the IPTp regimen and their neonates; $P$-values $\leq 0.05$ were considered significant.

\section{Results}

Of 536 women recruited, 516 were included in the analysis while 20 women who were lost to follow-up were excluded. Most of the participants (350) were recruited from the Federal Teaching Hospital while 186 were recruited from the Mile Four Catholic Maternity Hospital. The sociodemographic characteristics of the participants are shown in Table 1. The mean age of the participants was $28.09 \pm 4.78$ years. The following means were also obtained: gestational age at booking (21.80 \pm 6.93 weeks); gestational age at delivery (38.68 \pm 2.16 weeks); frequency of antenatal visits throughout the pregnancy (5.47 \pm 3.11$)$; dose of IPTp received (1.63 \pm 0.55$)$, and birth weight of babies delivered $(3,173.81 \pm 542.96 \mathrm{~g})$.

Table 2 summarizes the use and adherence to IPTp as well as reasons why women did not adhere to prescribed IPTp. Of the $35.4 \%$ (130/367) of study participants who received only one dose of IPTp, 68.8\% (89/130) failed to take subsequent doses for individual reasons ranging from not having money to pay for drugs (31.5\%), not feeling that they needed the drug $(16.9 \%)$ to forgetfulness $(12.3 \%)$ and first dose caused weakness $(7.7 \%)$.

Approximately $82 \%$ of participants who received IPTp had their first dose between 16-24 weeks gestation while $17.5 \%$ had their first dose between 24-28 weeks The second dose was given to $82.1 \%$ between $28-34$ weeks of gestation while only $17.9 \%$ had their second dose before 28 weeks gestation. The study hospitals did not have strict policies on DOT, and only 5\% (18/367) of parturients who took IPTp reported taking IPTp under the observation of the nursing staff. Patient-related reasons for not taking IPTp under DOT included not being told to do so (26\%), not having money to purchase the drug in the hospital (15\%), constraints of long waiting time (18\%), no reason $(3 \%)$. Facility-related reasons included inability of facilities to provide clean drinkable water $(20 \%)$, no staff to supervise the process $(10 \%)$, heavy patient load $(6 \%)$.

Table 3 summarizes the relationship of the occurrences of malaria in pregnancy and neonatal malaria to adherence to prescribed intermittent preventive treatment for malaria. Of the $79.8 \%$ who did not have clinical malaria during their pregnancies, approximately $64 \%$ included those who had at least two doses of IPTp. All those who had clinical malaria despite 
Table I Sociodemographic characteristics of participants according to uptake of IPTp-SP

\begin{tabular}{|c|c|c|c|c|}
\hline $\begin{array}{l}\text { Sociodemographic } \\
\text { variable }\end{array}$ & $\begin{array}{l}\text { Number of } \\
\text { participants } \\
n=516(\%)\end{array}$ & $\begin{array}{l}\text { Participants who } \\
\text { had no IPTp-SP } \\
n=\mid 49(\%)\end{array}$ & $\begin{array}{l}\text { Participants who } \\
\text { had } \geq \text { one dose of } \\
\text { IPTp-SP } n=367(\%)\end{array}$ & $P$-value \\
\hline \multicolumn{5}{|l|}{ Age (years) } \\
\hline$<20$ & $25(4.9)$ & $15(10.1)$ & $10(2.7)$ & \\
\hline $20-29$ & $310(60.0)$ & $66(44.3)$ & $244(66.5)$ & 0.02 \\
\hline $30-39$ & $178(34.5)$ & $65(43.6)$ & $113(30.8)$ & \\
\hline$\geq 40$ & $3(0.6)$ & $3(2.0)$ & $0(0)$ & \\
\hline \multicolumn{5}{|l|}{ Parity } \\
\hline$\leq 1$ & $233(45.2)$ & $33(22.1)$ & $200(54.5)$ & \\
\hline $2-4$ & $267(5 \mid .7)$ & $102(68.5)$ & $165(44.9)$ & $<0.000$ I \\
\hline$\geq 5$ & $16(3.1)$ & $14(9.4)$ & $2(0.5)$ & \\
\hline \multicolumn{5}{|l|}{ Educational status } \\
\hline Primary or less & $|2|(23.2)$ & $66(44.3)$ & $57(14.9)$ & $<0.0001$ \\
\hline Secondary or higher & $395(76.6)$ & $83(55.7)$ & $312(85.1)$ & \\
\hline \multicolumn{5}{|l|}{ Marital status } \\
\hline Married & 501 (97.I) & I36 (91.3) & $365(99.5)$ & 0.02 \\
\hline Single & $15(2.9)$ & $13(8.7)$ & $2(0.5)$ & \\
\hline \multicolumn{5}{|l|}{ Residence } \\
\hline Urban & $186(36.0)$ & $69(46.3)$ & 117 (31.9) & 0.04 \\
\hline Rural & $330(64.0)$ & $80(54.7)$ & $250(68.1)$ & \\
\hline
\end{tabular}

Abbreviation: IPTP-SP, intermittent preventive treatment for malaria with sulphadoxine-pyrimethamine.

IPTp had only one dose of IPTp despite booking in the second trimester. Malaria in pregnancy occurred significantly more in women who failed to adhere to prescribed IPTp than in those who adhered (24.6\% versus $14.3 \%$; RR $=2.53$;

Table 2 Use of IPTp, doses received, and reasons for nonadherence

\begin{tabular}{|c|c|}
\hline & $\begin{array}{l}\text { Number } \\
\text { of women (\%) }\end{array}$ \\
\hline \multicolumn{2}{|l|}{ Received IPTp } \\
\hline Yes & $367(7 \mid .2)$ \\
\hline No & $149(28.8)$ \\
\hline \multicolumn{2}{|l|}{ Number of doses of IPTp received $(n=367)$} \\
\hline One & $130(35.4)$ \\
\hline Two & $219(59.7)$ \\
\hline Three & $18(4.9)$ \\
\hline \multicolumn{2}{|l|}{ Reasons for not receiving IPTp } \\
\hline \multicolumn{2}{|l|}{ No IPTp at all $(n=\mid 49)$} \\
\hline Did not see the need & $49(32.9)$ \\
\hline Preferred herbs & $82(55.0)$ \\
\hline Religious reasons & $18(12.1)$ \\
\hline \multicolumn{2}{|l|}{ Failed to take subsequent doses $(n=130)$} \\
\hline Not prescribed & $40(31.0)$ \\
\hline Individual reasons & $89(68.8)$ \\
\hline $\begin{array}{l}\text { Did not have money when it was } \\
\text { prescribed }\end{array}$ & $4 I(31.5)$ \\
\hline $\begin{array}{l}\text { Did not know it was that important after } \\
\text { taking the first dose }\end{array}$ & $22(16.9)$ \\
\hline Simply forgot & $16(12.3)$ \\
\hline $\begin{array}{l}\text { First dose caused weakness and feeling of } \\
\text { ill health }\end{array}$ & $10(7.7)$ \\
\hline Allergy & I (0.2) \\
\hline
\end{tabular}

Abbreviation: IPTp, intermittent preventive treatment.
95\% confidence interval $[\mathrm{CI}] 2.10,3.04 ; P<0.001)$. Similarly, neonatal malaria occurred significantly more in neonates whose mothers did not receive IPTp compared to those whose mothers received at least one dose of IPTp (7.4\% versus $3.4 \%$; $\mathrm{RR}=1.38 ; 95 \% \mathrm{CI} 0.93,2.05 ; P=0.003$ ).

Low birth weight (LBW) occurred significantly more in live born singleton neonates delivered by women who did not receive IPTp at all compared to neonates born to women who took at least one dose of IPTp (10.5\% versus 3.7\%; $\mathrm{RR}=2.84 ; 95 \%$ CI $1.4,7.2 ; P<0.001)$.

During the study period, still birth accounted for $5 \%$ of perinatal deaths, of these $3.6 \%$ and $1.4 \%$ were fresh still birth and macerated still birth, respectively. More than half $(62.8 \%)$ of parturients who had still birth also had malaria in their third trimester. About $6.9 \%$ of neonates delivered by the participants who did not take IPTp had newborn special care unit admissions: $17.4 \%$ of these were due to neonatal malaria.

\section{Discussion}

Although the uptake of and adherence to use of IPTp-SP was assessed predominantly by self-reports by pregnant women and less by DOT, it would appear that awareness and uptake of IPTp was high in the sample of pregnant women studied. However, at a possible uptake level of approximately $72 \%$, the uptake of IPTp in this study was less than the finding in central Mozambique, where $92.5 \%$ of women received one or more doses of IPTp. ${ }^{14}$ The uptake level in this study 
Table 3 Comparison of maternal and neonatal malaria with use and nonuse of IPTP

\begin{tabular}{|c|c|c|c|c|}
\hline & IPTp $(n=367)$ & No IPTp $(n=149)$ & RR (95\% Cl) & \multirow[t]{2}{*}{$P$-value } \\
\hline & n (\%) & n (\%) & & \\
\hline \multicolumn{5}{|c|}{ Maternal $(n=5 \mid 6)$} \\
\hline Malaria & $74(20.2)$ & $127(85)$ & $2.53(2.10-3.04)$ & $<0.00 I^{*}$ \\
\hline No malaria & $293(79.8)$ & $22(15)$ & & \\
\hline \multicolumn{5}{|c|}{ Neonate $(n=5 \mid 6)$} \\
\hline Malaria & $12(3.4)$ & II (7.4) & $1.38(0.93-2.05)$ & $0.003 *$ \\
\hline No malaria & $355(96.6)$ & $138(92.6)$ & & \\
\hline
\end{tabular}

Note: *Represents statistically significant.

Abbreviations: $\mathrm{Cl}$, confidence interval; IPTP, intermittent preventive treatment; RR, risk ratio.

was also less than the target of $100 \%$ set by the Roll Back Malaria Partnership. ${ }^{1,2,15}$ Given that the WHO recommends monthly doses of SP after the first trimester and up to the time of delivery, the finding of an average of one and a half doses of IPTp-SP for an average of about five antenatal visits per woman showed that adherence to IPTp-SP was suboptimal. Also, the fact that the average of five antenatal visits per woman was not enough to ensure adherence to subsequent doses of IPTp-SP in this study suggests that better implementation of the policy for IPTp-SP is needed.

This study suggests that the role of health workers in the prescription and administration of IPTp could be critical to patients' adherence to its use during pregnancy. In this study, $31 \%$ of women did not receive subsequent doses of IPTp following the initial dose because the medications were not prescribed. Also, several health facility-related challenges were described to explain why IPTp was generally not administered using the recommended WHO model of DOT. Under DOT, SP for IPTp should be taken in the antenatal clinic under the direct observation of the care provider to increase uptake. However, for reasons such as lack of staff, lack of water, and heavy patient loads, health workers in the two facilities could not implement DOT. These reasons are similar to those found in the study in Mozambique. ${ }^{14}$

This study also found that malaria in pregnancy occurred significantly more in women who failed to adhere to prescribed IPTp than in those who complied. Only $20.2 \%$ of those who received at least one dose of IPTp had malaria compared to $85 \%$ of those who received no IPTp. The prevalence level of malaria of $20 \%$ is similar to $17.6 \%$ reported in Uganda in $2008 .{ }^{16}$ The high preponderance of clinical malaria among those who failed to adhere to prescribed IPTp further underlies the increased risk of malaria and its maternal-fetal complications when IPTp-SP is not used.

This study also found a higher prevalence of LBW recorded for women who did not receive IPTp compared to those who had three doses. This finding agrees with the findings of Le Hesran et al. ${ }^{17}$ Although this study did not control for other factors that impact fetal weight, it is possible that our findings can be explained by the known association between malaria in pregnancy and LBW, due mainly to the effect of placental parasitemia on fetal nutrition.

Another notable finding from this study was the fact that there was a high preponderance of women who had malaria in the third trimester of pregnancy among those who delivered stillborn babies. Given that most of those who had malaria in pregnancy were those who failed to adhere to the use of IPTp, this finding lends support to the deleterious effect of nonadherence to malaria prophylaxis to fetal well-being. Further evidence from this study on the harmful effect of nonadherence to malaria prophylaxis was the fact that neonatal malaria contributed about $18 \%$ of neonatal intensive care admissions among those who did not adhere to prescribed IPTp.

The major strength of this study was the prospective nature, which enabled direct collection of data. The drawbacks include the inability to use DOT to establish actual use of IPTp for most patients. Reliance on self-reported use of IPTp is liable to misinformation. These drawbacks were addressed by counselling participants who report failure to take IPTp while not attracting any form of punishment whatsoever.

In conclusion, the potential coverage rate of IPTp in the study area is still below the global and national recommendation, with over one half of women either failing to take IPTp at all or adhere to subsequent doses of prescribed IPTp. The preponderance of clinical maternal malaria, neonatal malaria, and LBW among defaulters of IPTp further underlies the need to strengthen the use of prophylactic malaria drugs in this area. Tackling malaria in pregnancy using well designed IPTp programs with strict adherence to the DOT policy, which are now an integral part of WHO's "Making Pregnancy Safer" initiative, should be part of routine ANC. These in addition to other malaria preventive interventions should contribute positively to the attainment of the millennium development 
goals 4 (reduce child mortality by 2/3), 5 (improve maternal mortality by $3 / 4$ ), and 6 (combat human immunodeficiency virus/acquired immunodeficiency syndrome, malaria, and other diseases) in the study area as in most malaria-endemic countries of sub-Sahara Africa.

We further recommend that since malaria is a public health issue, there should be sustained widespread public enlightenment about malaria, IPTp and its benefits using communication media that reaches the vast majority in rural and remote communities, in the language the people speak and understand. Avenues for capacity building by training and retraining health care providers on issues related to malaria and IPTp should be created. SP should also be supplied free to pregnant women by the government to facilitate the implementation of DOT.

\section{Disclosure}

The authors report no conflicts of interest in this work.

\section{References}

1. Roll Back Malaria Partnership. Focus on Nigeria. Progress and Impact Series, Country Reports Number 4. Geneva: Roll Back Malaria Partnership; 2012. Available from: http://www.rbm.who. int/ProgressImpactSeries/docs/report11-en.pdf. Accessed January 26, 2014.

2. Federal Ministry of Health. A Road Map for Impact on Malaria in Nigeria. A 5-year strategic plan (2006-2010). Abuja: Federal Ministry of Health, National Malaria Control Programme. Available at: http:// www.rbm.who.int/countryaction/nsp/nigeria.pdf. Accessed January 25, 2014.

3. Steketee RW, Nahlen BL, Parise ME, Menendez C. The burden of malaria in pregnancy in malaria-endemic areas. Am J Trop Med Hyg. 2001;64(Suppl 1-2):28-35.

4. Dellicour S, Tatem AJ, Guerra CA, Snow RW, ter Kuile FO. Quantifying the number of pregnancies at risk of malaria in 2007: a demographic study. PLoS Med. 2010;7(1):e1000221.

5. Wagbatsoma VA, Omoike BI. Prevalence and prevention of malaria in pregnancy in Edo State, Nigeria. Afr J Reprod Health. 2005;12(3):49-58.
6. Chukwuocha UM, Dozie IN, Chukwuocha AN. Malaria and its burden among pregnant women in parts of the Niger Delta area of Nigeria. Asian Pac J Reprod. 2012;1(2):147-151.

7. World Health Organization. A strategic framework for malaria prevention and control during pregnancy in the African region [webpage on the Internet]. Geneva: World Health Organization; 2004. Available from: http://www.who.int/malaria/publications/atoz/afr_mal_04_01/en/. Accessed December 20, 2013.

8. World Health Organization. WHO policy brief for the implementation of intermittent preventive treatment of malaria in pregnancy using sulfadoxine-pyrimethamine (IPTp-SP) [webpage on the Internet]. Geneva: World Health Organization; 2013 [updated January 2014]. Available from: http://www.who.int/malaria/publications/atoz/policy_brief_iptp_ sp_policy_recommendation/en/. Accessed February 22, 2014.

9. Federal Ministry of Health, Nigeria. National guidelines and strategies for malaria prevention and control during pregnancy [webpage on the internet]. Abuja: Federal Ministry of Health, Nigeria; 2010. Available from: http://www.rbm.who.int/countryaction/nsp/nigeria.pdf. Accessed April 3, 2014.

10. Ugwu EO, Ezechukwu PC, Obi SN, Ugwu AO, Okeke TC. Utilization of insecticide treated nets among pregnant women in Enugu, South Eastern Nigeria. Niger J Clin Pract. 2013;16(3):292-296.

11. Onwujekwe O, Uzochukwu B, Ezumah N, Shu E. Increasing coverage of insecticide-treated nets in rural Nigeria: implications of consumer knowledge, preferences and expenditures for malaria prevention. Malar J. 2005;4(1):29.

12. Ukibe SN, Mbanugo JI, Ukibe NR, Ikeakor LC. Level of awareness and use of insecticide treated bed nets among pregnant women attending antenatal clinics in Anambra State, South East Nigeria. J Public Health Epidemiol. 2013;5(9):391-396.

13. Aluko JO, Oluwatosin AO. Utilization of insecticide treated nets during pregnancy among postpartum women in Ibadan, Nigeria: a crosssectional study. BMC Pregnancy Childbirth. 2012;12:21.

14. Brentlinger PE, Dgedge M, Correia MA, et al. Intermittent preventive treatment of malaria during pregnancy in central Mozambique. Bull World Health Organ. 2007;85(11):873-879.

15. Roll Back Malaria Partnership. Global malaria action plan for a malariafree world [webpage on the Internet]. Geneva: Roll Back Malaria Partnership. Available from: http://www.rollbackmalaria.org/gmap/1-2. html. Accessed February 23, 2014.

16. Mbonye AK, Bygbjerg IC, Magnussen P. Intermittent preventive treatment of malaria in pregnancy: a new delivery system and its effect on maternal health and pregnancy outcomes in Uganda. Bull World Health Organ. 2008;86(2):93-100.

17. Le Hesran JY, Cot M, Personne P, et al. Maternal placental infection with Plasmodium falciparum and malaria morbidity during the first 2 years of life. Am J Epidemiol. 1997;146(10):826-831.
Patient Preference and Adherence

\section{Publish your work in this journal}

Patient Preference and Adherence is an international, peer-reviewed, open access journal that focusing on the growing importance of patient preference and adherence throughout the therapeutic continuum. Patient satisfaction, acceptability, quality of life, compliance, persistence and their role in developing new therapeutic modalities and compounds to optimize

\section{Dovepress}

clinical outcomes for existing disease states are major areas of interest for the journal. This journal has been accepted for indexing on PubMed Central. The manuscript management system is completely online and includes a very quick and fair peer-review system, which is all easy to use. Visit http://www. dovepress.com/testimonials.php to read real quotes from published authors. 